\title{
Nonlinear response in the cumulant expansion for core-level photoemission
}

\author{
Marilena Tzavala, ${ }^{1,2,3}$ J. J. Kas, ${ }^{1,3}$ Lucia Reining $\odot,{ }^{2,3, *}$ and J. J. Rehr $\circledast^{1,3, \dagger}$ \\ ${ }^{1}$ Department of Physics, University of Washington, Seattle, Washington 98195-1560, USA \\ ${ }^{2}$ Laboratoire des Solides Irradiés, École Polytechnique, CNRS, CEA/DRF/IRAMIS, \\ Institut Polytechnique de Paris, F-91128 Palaiseau, France \\ ${ }^{3}$ European Theoretical Spectroscopy Facility (ETSF)
}

(Received 4 May 2020; revised 3 July 2020; accepted 6 July 2020; published 27 July 2020)

\begin{abstract}
Most currently used approximations for the one-particle Green's function $G$ in the framework of many-body perturbation theory, such as Hedin's GW approximation or the cumulant GW $+\mathrm{C}$ approach, are based on a linear-response approximation for the screened interaction $W$. The extent to which such a hypothesis is valid and ways to go beyond have been explored only very little. Here we show how to derive a cumulant Green's function beyond linear response from the equation of motion of the Green's function in a functional derivative formulation. The results can be written in a compact form, which opens the possibility to calculate the corrections in a first-principles framework using time-dependent density functional theory. In order to illustrate the potential importance of the corrections, numerical results are presented for a model system with a core level and two valence orbitals.
\end{abstract}

DOI: 10.1103/PhysRevResearch.2.033147

\section{INTRODUCTION}

Core-level x-ray photoemission spectroscopy (XPS) is a sensitive probe of correlation properties in condensed matter [1]. At high photon energies, where extrinsic and interference effects due to scattering of the outgoing photoelectron can be neglected, the XPS photocurrent is directly related to the spectral function associated with the core-hole Green's function. The importance of many-body effects for spectra related to core excitations has long been recognized, and the corresponding research has a rich history, going back at least to the 1920s [2-4]. Of particular interest are edge singularities and asymmetric line shapes. These could be explained by the coupling between the core hole and the valence electrons using studies based on model Hamiltonians, e.g., in the seminal works of Mahan, Nozières, and De Dominicis, and co-workers [1,5-9]. Several exact and approximate results were obtained, for example assuming the interaction potential is separable [5]. Another commonly used assumption is the absence of interaction between the valence electrons themselves, which implies that their scattering from the core-hole potential leads to the excitation of independent electron-hole pairs. In the same framework, the core-hole problem was treated by solving two coupled Bethe-Salpeter equations for core and valence electrons to lowest order in a parquet approximation [6], to which self-consistency in self-energy and vertex were added in Ref. [7]. In the subsequent paper of the same series [8],

\footnotetext{
*lucia.reining@polytechnique.fr

†jjr@uw.edu

Published by the American Physical Society under the terms of the Creative Commons Attribution 4.0 International license. Further distribution of this work must maintain attribution to the author(s) and the published article's title, journal citation, and DOI.
}

an effective one-body approach was proposed, based on the calculation of the transient response to the sudden creation of a core hole. This was complemented by Langreth [10] with a more compact derivation, and in Ref. [11] by an alternative approach based on a finite number of electrons in a box, which validated the approximation of a separable potential and added numerical illustrations.

The picture of excitations created by the sudden appearance of the core hole is also naturally reflected in fermionboson coupling Hamiltonians, where the fermion refers to a deep core orbital, and the bosons are electron-hole excitations, plasmons [12,13], or phonons [14-17]. Remarkably, for a single fermion with linear coupling to a dispersing boson, the model can be solved exactly [18-20]. The fermionic spectral function then consists of a quasiparticle peak followed by a Poisson series of satellites. For increasing coupling strength the quasiparticle loses increasing weight to the satellites, and the envelope of the satellite spectrum becomes a Gaussian in shape. For this model, this exact solution is equivalent to the spectral function of the second-order (in the coupling constant) cumulant Green's function [1,15]. For more than one fermion energy and/or for higher-order coupling to bosons, the cumulant solution is not exact [15]. Nevertheless, since it contains the essential physics of the system responding by bosonic excitations to the creation of an additional electron or hole, it is often a good approximation that is widely used, in particular for the core-hole problem [21-27].

Of course the electron-boson picture is just another way to look at the old problem of many-body effects in core spectroscopy, but it highlights three questions that one might ask, namely,

(i) How well does a Hamiltonian with linear electronboson coupling describe the real problem?

(ii) Which excitations should be contained in the bosons?

(iii) Is the second-order cumulant solution good enough for real applications or, if not, how can one go beyond? 
Heretofore these questions have not found a definite answer, not least because the domain of application of the cumulant approach in condensed matter is very wide, including, for example, coupling to phonons [16,28] and valence electron spectroscopy [29-40].

Analogous questions arise in an at-first-sight, different framework, which is the calculation of Green's functions from a Dyson equation, where interaction effects are contained in the self-energy, the integral kernel of the equation [41]. This is the framework now commonly adopted in first-principles calculations. Currently the most widely used approximation is Hedin's GW approximation (GWA) [42], where the self-energy is approximated as the product of the one-electron Green's function $G$ and the screened Coulomb interaction $W$ to first order. Indeed, one can view the GW approximation as an approximate solution of the electron-boson problem [1], where the bosonic excitations are the excitations contained in $W$. Also in this case, since the coupling is linear, the bosons contain only a selection of excitations (spin-flip excitations, for example, are neglected), and the problem is solved only approximately. While the GWA has been very successful for the calculation of quasiparticle energies, it typically gives a poor description of satellite spectra [43]. This shortcoming can be traced to the third of the above questions - the approximation used in the solution of the Hamiltonian problem-since this approximation leads to problems such as the appearance of a spurious plasmaron solution. This can be seen in the GW solution of the exactly solvable model [1]. Changing from the GWA to a cumulant Green's function leads to a significant improvement without much additional effort, since the same boson appears through $W$ and the electron-boson problem is solved more accurately. The GW plus cumulant $(\mathrm{GW}+\mathrm{C})$ approach for the one-electron Green's function [29,32] has recently become a popular first-principles method to describe spectral functions, including satellite series. Here, the satellites are mostly due to plasmons [36], the dominant excitations in $W$.

The success of the $\mathrm{GW}$ and $\mathrm{GW}+\mathrm{C}$ approximations may seem surprising in view of the relative simplicity of these approximations being linear in $G$ and $W$. While it is physically reasonable, e.g., in many simple semiconductors, that plasmonic excitations should represent the dominant boson, it is less obvious that the physics is sufficiently described by linear response. This is especially evident in systems with few electrons, or when the removal or addition orbital is very localized, where one might expect that response terms of higher order should play a role. These terms are not contained in the electron-boson model with linear coupling, because the boson itself is fixed and does not respond to the excitation; analogously, they are contained in neither $\mathrm{GW}$ nor $\mathrm{GW}+\mathrm{C}$ because $W$ is calculated in the ground state, in the presence of the original $N$ electrons. Although nonlinear corrections are implicit in the diagrammatic expression of Langreth [18], a more detailed study of vertex corrections suggested that their effect on plasmon excitations is small $[22,44]$. The fact that nonlinear screening should be present and should show up in a cumulant solution was also pointed out by Mahan [5], who calculated the leading correction to the standard secondorder (in the coupling constant) linear-response cumulant. In that work the valence electrons that respond to a core-hole excitation are described by an independent-electron picture, and even so, going to yet higher orders turned out to be too complicated. While these pioneering works, as well as the seminal solution of Nozières and De Dominicis [8], trace a way to go towards the inclusion of nonlinear screening effects, these model approaches are not directly transferable to first-principles calculations for several reasons: (i) because of the approximations involved from the very beginning on the interaction potential; (ii) because of the absence of interaction between valence electrons, which would lead to a poor description of screening in the absence of plasmons; and (iii) because it is not clear how an expansion that is order-byorder concerning the response functions (i.e., linear response, second-order response, etc.) would converge. Progress in several directions has been made, such as a better description of screening $[45,46]$, higher-order cumulant solutions of the electron-boson model, such as in Refs. [15,47,48], the derivation of higher-order correlation functions from the MahanNozières-De Dominicis (MND) or electron-boson Hamiltonians [49], or more recently with the description of nonlinear electron-boson couplings in Ref. [50], and in the equationof-motion, coupled-cluster method [51,52]. However, there is still a gap to be filled concerning the $a b$ initio calculation of spectral functions.

The present work aims at developing a robust, firstprinciples derivation of nonlinear screening effects in a cumulant Green's function. While we do not claim to invent new physics here, our derivation has the advantage of being compact, and situated within the framework now commonly used in the $a b$ initio community. Our approach does not depend on a contact or on separability approximations for the interaction potential. Moreover, the approach highlights the underlying physics, which is crucial if one wishes to understand when nonlinear effects are important, and where should be the limits of their applicability. Finally, it allows us to propose a way to put the equations into practice, by combining the many-body perturbation formalism with timedependent density functional theory (TDDFT) [53]. In this way, an order-by-order expansion of the response is avoided, and the problem of calculating the effects of screening on the Green's function is separated from that of the calculation of the screening itself. Contrary to numerous previous works on core spectroscopy, we are not so much interested in asymmetry of line shapes but rather in the satellites, for which the standard second-order linear response cumulant approach is now a well-established first-principles approach and convenient starting point.

The paper is organized as follows: The background concerning the formalism and the GW approximation is contained in Sec. II. Next, we derive the core-hole cumulant Green's function in its various approximations in Sec. III, and we analyze the results in Sec. IV. Numerical results for an illustrative model are given in Sec. V, and a short conclusion is given in Sec. VI.

\section{BACKGROUND}

Beginning with its equation of motion following the approach of Martin and Schwinger [54], the one-body Green's function can be described by a functional differential equation 
which is often referred to as the Kadanoff-Baym equation [55]. The equation was initially derived for temperaturedependent, nonequilibrium quantum systems. However, it can also be used to create the diagrams describing equilibrium and/or zero-temperature physics [43], as we do here. In this formalism, the fully interacting propagator $G$ is given by

$$
\begin{aligned}
G(12)= & G_{0}(12)-i G_{0}(1 \overline{1}) v(\overline{1} \overline{3}) G\left(\overline{3}^{3}{ }^{+}\right) G(\overline{1} \overline{2}) \\
& +i G_{0}(1 \overline{1}) v(\overline{1} \overline{3}) \frac{\delta G(\overline{1} \overline{2})}{\delta u\left(\overline{3}^{+}\right)},
\end{aligned}
$$

where $G_{0}$ is the noninteracting propagator, $v(12)=\delta\left(t_{1}-\right.$ $\left.t_{2}\right) /\left|\mathbf{r}_{1}-\mathbf{r}_{2}\right|$ is the Coulomb interaction, and $u(3)$ is a local, time-dependent external potential that simulates interaction effects due to the propagation of particles and will be taken to zero at the end of the calculation. Here and below we employ the usual notation of an integer for a set of space, spin, and time variables $\left[1 \rightarrow\left(r_{1}, \sigma_{1}, t_{1}\right)\right]$, and bars for variables that are integrated over: $f(\overline{1}) g(\overline{1}) \equiv \int d 1 f(1) g(1)$. All quantities that can be derived from $G$ or $G_{0}$ are therefore functionals of $u$. For simplicity the variational parameter $u(\mathbf{r}, t)$ will be regarded as nonzero and implicit in the Green's function and related quantities, unless otherwise stated. The classical Hartree term (the second term on the right) depends on the interacting density as given by the diagonal part of the propagator $n(1)=-i G\left(11^{+}\right)$, where $1^{+} \equiv \lim _{\eta \rightarrow 0}\left(\mathbf{r}_{1}, \sigma_{1}, t_{1}+\eta\right)$, $\eta>0$. The last term contains the functional derivative of $G$, which accounts for exchange and correlation effects. This term turns Eq. (1) into a first-order nonlinear functional differential equation with respect to $u$. Introducing the total classical potential $u^{H}$, one obtains a set of coupled equations for $u^{H}$ and $G$ :

$$
\begin{gathered}
u^{H}(1)=u(1)+v(1 \overline{3}) n(\overline{3}), \\
G^{H}(12)=G_{0}(12)+G_{0}(1 \overline{4}) u^{H}(\overline{4}) G^{H}(\overline{4}, 2), \\
G(12)=G^{H}(12)+G^{H}(1 \overline{1}) v(\overline{1} \overline{3}) \frac{\delta G(\overline{1} 2)}{\delta u\left(\overline{3}^{+}\right)} .
\end{gathered}
$$

In extended systems, screening plays an important role. For this reason, is is convenient to rewrite the functional derivative using the chain rule with respect to the classical potential,

$$
G(12)=G^{H}(12)+i G^{H}(1 \overline{1}) W(\overline{1} \overline{4} ; u) \frac{\delta G(\overline{1} 2)}{\delta u^{H}\left(\overline{4}^{+}\right)},
$$

where

$$
W(14 ; u) \equiv v(1 \overline{3}) \frac{\delta u^{H}(4)}{\delta u(\overline{3})} .
$$

Here the screened potential $W$ is a functional of the external potential, such that the equation remains exact.

Often, the dependence of $W$ on $u$ is neglected. The solution of this linear-response version of Eq. (5) in a simple model has been discussed in Ref. [56]. In general, however, even in the linear-response approximation, the equation cannot be solved exactly. Therefore, the functional derivative on the righthand side is usually approximated such that the limit $u \rightarrow 0$ can be taken directly. For example, one of the most widely used approximations for the Green's function is Hedin's GW approach [42], which is obtained by approximating the functional derivative as

$$
\frac{\delta G(12)}{\delta u^{H}(4)} \approx G(14) G(42) .
$$

Then the $u \rightarrow 0$ limit can be taken, and one has a Dyson equation for $G$,

$$
G(12)=G^{H}(12)+G^{H}(1 \overline{3}) \Sigma_{\mathrm{xc}}^{G W}(\overline{3} \overline{4}) G(\overline{4} 2),
$$

with

$$
\Sigma_{\mathrm{xc}}^{G W}(14) \equiv i G(14) W(14 ; u \rightarrow 0) .
$$

The GWA has been very successful for the calculation of quasiparticle energies. However, a major shortcoming is its poor treatment of the satellite part of electron addition or removal spectra $[29,32,36,43]$. Calculations based on a cumulant Green's function, which yields a much much better satellite spectrum, have been found to be an advantageous alternative. The cumulant approach, which avoids the approximation in Eq. (7) that leads to the GW self-energy, is discussed in the following section.

\section{CORE-HOLE CUMULANT}

In order to describe the photoemission spectra from core levels, we need the projection of the Green's function on the core orbital, $G_{c c}$. Its associated spectral function simulates the core photoemission spectrum, especially at high photon energies, where the photoelectron and associated effects of extrinsic losses can be ignored. We start by expressing Eq. (4) for $G$ in a basis of single-particle orbitals,

$$
G_{i j}\left(t_{1} t_{2}\right)=G_{i j}^{H}\left(t_{1} t_{2}\right)+G_{i m}^{H}\left(t_{1} t_{\overline{1}}\right) v_{m n k l} \frac{\delta G_{n j}\left(t_{\overline{1}} t_{2}\right)}{\delta u_{k l}\left(t_{\overline{1}}^{+}\right)},
$$

where here and throughout this paper repeated indices are summed over. We used for this basis transformation the chain rule

$$
\begin{aligned}
\frac{\delta G_{n j}\left(t_{1} t_{2}\right)}{\delta u\left(r_{3}, t_{1}^{+}\right)} & =\frac{\delta G_{n j}\left(t_{1} t_{2}\right)}{\delta u_{k l}\left(t_{1}^{+}\right)} \frac{\delta u_{k l}\left(t_{1}^{+}\right)}{\delta u\left(r_{3}, t_{1}^{+}\right)} \\
& =\frac{\delta G_{n j}\left(t_{1} t_{2}\right)}{\delta u_{k l}\left(t_{1}^{+}\right)} \phi_{k}^{*}\left(r_{3}\right) \phi_{l}\left(r_{3}\right),
\end{aligned}
$$

with $u_{k l}\left(t_{1^{+}}\right) \equiv \int d r_{3} u\left(r_{3}\right) \phi_{k}^{*}\left(r_{3}\right) \phi_{l}\left(r_{3}\right)$, spin is understood to be treated analogous to space, and matrix elements of the bare Coulomb interaction are defined as $v_{m n k l}=$ $\int d r d r^{\prime} \phi_{m}^{\star}(r) \phi_{n}(r) \phi_{k}^{\star}\left(r^{\prime}\right) \phi_{l}\left(r^{\prime}\right) /\left|r-r^{\prime}\right|$. We now make the approximation that the core hole is decoupled from all other orbitals, except for the screening of the interaction, which is due to the valence electrons. This decoupling approximation is physically reasonable for the case of localized electrons, such as a deep-core state, which has little overlap with the valence electrons. Moreover, we limit our discussion to the case of an $s$ state ( $K$ shell), where spin-degeneracy and spin-orbit effects are not relevant. For this case, we may suppose that the interacting and the Hartree Green's functions $G$ and $G^{H}$ do not have matrix elements linking the core state to another core or valence state, so $G_{c i}=\delta_{c i} G_{c c}$ and $G_{c i}^{H}=\delta_{c i} G_{c c}^{H}$, and Eq. (11) becomes

$$
G_{c c}\left(t_{1} t_{2}\right)=G_{c c}^{H}\left(t_{1} t_{2}\right)+i G_{c c}^{H}\left(t_{1} \bar{t}_{3}\right) v_{c c k l} \frac{\delta G_{c c}\left(\bar{t}_{3} t_{2}\right)}{\delta u_{k l}\left(\bar{t}_{3}^{+}\right)} .
$$


In principle, before the equation is solved and the timedependent external potential is set to zero, the system is out of equilibrium and cannot be described by equilibrium Green's functions. The physical solution for all (vanishing or nonvanishing) $u$ may be obtained, e.g., using Green's functions on the Keldysh contour [57]. However, when, as is the case here, only the final solution for vanishing $u$ is of interest, other constructions are possible, such as for example, the auxiliary Green's function introduced by Hedin [42]. The situation simplifies when the mixing of forward and backward propagation in time is neglected. In the present work, we make this approximation, so for a core level we only retain the contributions that correspond to the propagation of a hole, i.e., $t_{2}>\bar{t}_{3}>t_{1}$. This allows us to make the ansatz that the interacting propagator is proportional to $G^{H}$, i.e.,

$$
G_{c c}\left(t_{1} t_{2}\right)=G_{c c}^{H}\left(t_{1} t_{2}\right) F\left(t_{1} t_{2}\right) .
$$

With the identity $\frac{\delta G}{\delta U}=\frac{\delta G^{H}}{\delta U} F+G^{H} \frac{\delta F}{\delta U}$, we obtain

$$
\begin{aligned}
F\left(t_{1} t_{2}\right)= & +i \frac{G_{c c}^{H}\left(t_{1} \bar{t}_{1}\right)}{G_{c c}^{H}\left(t_{1} t_{2}\right)} \\
& \times v_{c c k l}\left[\frac{\delta G_{c c}^{H}\left(\bar{t}_{1} t_{2}\right)}{\delta u_{k l}\left(\bar{t}_{1}^{+}\right)} F\left(\bar{t}_{1} t_{2}\right)+G_{c c}^{H}\left(\bar{t}_{1} t_{2}\right) \frac{\delta F\left(\bar{t}_{1} t_{2}\right)}{\delta u_{k l}\left(\bar{t}_{1}^{+}\right)}\right] .
\end{aligned}
$$

As in the derivation of the GWA, we can now use the chain rule with the total classical potential. Moreover, as for $G$ we suppose that $G^{H}$ has no off-diagonal elements linking core and valence single-particle states, even when $u \neq 0$, which is exact when the wavefunctions do not overlap. This leads to

$$
\begin{aligned}
F\left(t_{1} t_{2}\right)= & 1+i \frac{G_{c c}^{H}\left(t_{1} \bar{t}_{1}\right)}{G_{c c}^{H}\left(t_{1} t_{2}\right)} \\
& \times\left[W_{c}\left(\bar{t}_{1}^{+} \bar{t}_{4} ; u\right) G_{c c}^{H}\left(\bar{t}_{1} \bar{t}_{4}\right) G_{c c}^{H}\left(\bar{t}_{4} t_{2}\right) F\left(\bar{t}_{1} t_{2}\right)\right. \\
& \left.+v_{c c k l} G_{c c}^{H}\left(\bar{t}_{1}, t_{2}\right) \frac{\delta F\left(\bar{t}_{1} t_{2}\right)}{\delta u_{k l}\left(\bar{t}_{1}^{+}\right)}\right]
\end{aligned}
$$

with the screened interaction $W_{c} \equiv W_{c c c c}$, where

$$
\begin{aligned}
W_{c}\left(t_{1}^{+} t_{4}\right) \equiv & W_{c c c c}\left(t_{1}^{+} t_{4}\right)=v_{c c c c} \delta\left(t_{4} t_{1}^{+}\right) \\
& +v_{c c k l} v_{c c k^{\prime} l^{\prime}} \frac{\delta n_{k l}\left(t_{4}\right)}{\delta u_{k^{\prime} l^{\prime}}\left(t_{1}^{+}\right)} .
\end{aligned}
$$

At this stage $W_{c}\left(t_{1} t_{4} ; u\right)$ still depends on the potential $u$, which has not yet been set to zero. Also note that Eq. (16) suggests a response of the density; however, one has to keep in mind that here we are not in a retarded framework, but only keep parts corresponding to the time ordering defined above.

Within our approximations, where we keep only one of the time orderings and neglect core-valence overlap, the Hartree core Green's function becomes $G_{c c}^{H}\left(t_{1} t_{2}\right)=$ $i \exp \left(-i \varepsilon_{c}^{0}\left(t_{1}-t_{2}\right)+i \int_{t_{1}}^{t_{2}} d \tau u_{c c}^{H}(\tau)\right) \theta\left(t_{2}-t_{1}\right)$. Therefore, integrals of Green's functions simplify, so

$$
\int_{-\infty}^{+\infty} d \bar{t}_{4} G_{c c}^{H}\left(\bar{t}_{1} \bar{t}_{4}\right) G_{c c}^{H}\left(\bar{t}_{4} t_{2}\right)=i G_{c c}^{H}\left(\bar{t}_{1} t_{2}\right) \int_{\bar{t}_{1}}^{t_{2}} d \bar{t}_{4}
$$

and

$$
\int_{-\infty}^{+\infty} d \bar{t}_{1} G_{c c}^{H}\left(t_{1} \bar{t}_{1}\right) G_{c c}^{H}\left(\bar{t}_{1} t_{2}\right)=i G_{c c}^{H}\left(t_{1} t_{2}\right) \int_{t_{1}}^{t_{2}} d \bar{t}_{1} .
$$

Consequently $G_{c c}^{H}\left(t_{1} t_{2}\right)$ factorizes and the factors cancel, so that

$$
\begin{aligned}
F\left(t_{1} t_{2}\right)= & 1-i \int_{t_{1}}^{t_{2}} d \bar{t}_{1} \int_{\bar{t}_{1}}^{t_{2}} d \tau W_{c}\left(\bar{t}_{1}^{+} \tau ; u\right) F\left(\bar{t}_{1} t_{2}\right) \\
& -v_{c c k l} \int_{t_{1}}^{t_{2}} d \bar{t}_{1} \frac{\delta F\left(\bar{t}_{1} t_{2}\right)}{\delta u_{k l}\left(\bar{t}_{1}^{+}\right)} .
\end{aligned}
$$

Next, we define $F$ in cumulant form,

$$
F\left(t_{1} t_{2}\right) \equiv e^{C\left(t_{1} t_{2}\right)},
$$

and take the derivative of Eq. (19) with respect to $t_{1}$. This yields a differential equation for the cumulant function $C\left(t t^{\prime}\right)$ :

$$
\partial_{t_{1}} C\left(t_{1} t_{2}\right)=i \int_{t_{1}}^{t_{2}} d \tau W_{c}\left(t_{1}^{+} \tau ; u\right)+v_{c c k l} \frac{\delta C\left(t_{1} t_{2}\right)}{\delta u_{k l}\left(t_{1}^{+}\right)} .
$$

With the boundary condition $C\left(t_{2} t_{2}\right)=0$, which preserves normalization of the spectral function, the integral equation for $C$ is given by

$$
\begin{aligned}
C\left(t_{1} t_{2}\right)= & -i \int_{t_{1}}^{t_{2}} d \tau^{\prime} \int_{\tau^{\prime}}^{t_{2}} d \tau W_{c}\left(\tau^{\prime+} \tau ; u\right) \\
& -v_{c c k l} \int_{t_{1}}^{t_{2}} d \tau^{\prime} \frac{\delta C\left(\tau^{\prime} t_{2}\right)}{\delta u_{k l}\left(\tau^{\prime+}\right)} .
\end{aligned}
$$

The last term of this expression is commonly neglected, so one can directly set the external potential $u$ to zero. That approximation yields the widely used expression for the cumulant in the linear-response approximation [36],

$$
\begin{aligned}
C^{0}\left(t_{1} t_{2}\right) & =-i \int_{t_{1}}^{t_{2}} d \tau^{\prime} \int_{\tau^{\prime}}^{t_{2}} d \tau W_{c}\left(\tau^{\prime+} \tau ; u\right) \\
& =-i \int_{t_{1}}^{t_{2}} d \tau \int_{t_{1}}^{\tau} d \tau^{\prime} W_{c}\left(\tau^{\prime+} \tau ; u\right) .
\end{aligned}
$$

For the core hole, together with Eq. (16) the expression can be interpreted as the integral over the variation of the Coulomb potential due the valence density at time $\tau$, induced in linear response of the system by the creation of a hole at time $\tau^{\prime}$. The response is causal with $\tau>\tau^{\prime}$, and the process is integrated over the interval $\left[t_{1}, t_{2}\right]$. Implicit in the evaluation of the cumulant $C^{0}$ is that only the positive frequency components of the Fourier transform $W_{c}(\omega)$ corresponding to lossy excitations are present in the linear-response approximation [18].

To go beyond linear response, one can iterate Eq. (22). The lowest-order corrections stem from the derivative of $C^{0}$, taking into account that $\delta W_{c} / \delta u \neq 0$. This causes a secondorder response function to appear in a contribution $C^{1}$ given by

$$
C^{1}\left(t_{1} t_{2}\right)=i v_{c c k l} \int_{t_{1}}^{t_{2}} d \tau \int_{t_{1}}^{\tau} d \tau^{\prime} \int_{t_{1}}^{\tau^{\prime}} d \tau^{\prime \prime} \frac{\delta W_{c}\left(\tau^{\prime} \tau ; u\right)}{\delta u_{k l}\left(\tau^{\prime \prime+}\right)} .
$$

This term contains the variation of the response of the valence density due to the density change caused by the linear response to the creation of the core hole. 
Higher-order corrections involve higher-order derivatives of $W_{c}$, and therefore higher-order nonlinear response functions. They should be evaluated from the density-density correlation functions in the ground state, i.e., before the removal of the core electron. The general form of the solution is thus given by

$$
C\left(t_{1} t_{2}\right)=\sum_{m=0} C^{m}\left(t_{1} t_{2} ; u=0\right)
$$

where the $m$ th contribution $C^{m}$ is obtained recursively using the relation

$$
C^{m+1}\left(t_{1} t_{2} ; u=0\right)=-\left.v_{c c k l} \int_{t_{1}}^{t_{2}} d \tau \frac{\delta C^{m}\left(\tau t_{2} ; u\right)}{\delta u_{k l}\left(\tau^{+}\right)}\right|_{u=0},
$$

with $C^{0}$ given by Eq. (23) in terms of $W_{c}$. This recursive formulation suggests that the series might in practice be truncated at a given order $n$. Once all orders of the correction have been found, the limit of $u \rightarrow 0$ is applied. The full recursive solution implied by Eq. (26) is the first main result of this paper. It may be seen as an extension of Mahan's approach in Ref. [5], which contains the first nonlinear response contribution. The key differences are a more general formulation such that (i) a separable potential is not needed for the derivation; (ii) the result is valid to infinite order in the response; and, most importantly, (iii) the valence electrons are in principle fully interacting. As we see below, the fact that the result is formulated in general terms of response functions rather than explicit sums over transitions allows us to benefit from the use of TDDFT for an efficient approximation to the nonlinear response.

\section{ANALYSIS}

\section{A. Effective interaction}

The leading cumulant $C^{0}\left(t t^{\prime}\right)$ in Eq. (23) and similarly the higher-order terms are double integrals of a two-time function over time. We can therefore express the cumulant in terms of a new function $w\left(\tau \tau^{\prime}\right) \equiv \sum_{m=0} w^{m}\left(\tau \tau^{\prime}\right)$, where $w^{0}\left(\tau \tau^{\prime}\right)=$ $W_{c}\left(\tau^{\prime} \tau\right)$, and $w^{m}$ stands for the order- $m$ correction in $W_{c}$. Each cumulant term is related to $w$ through the expression

$$
C^{m}\left(t_{1} t_{2}\right)=-i \int_{t_{1}}^{t_{2}} d \tau \int_{t_{1}}^{\tau} d \tau^{\prime} w^{m}\left(\tau \tau^{\prime}\right)
$$

A recursive relation, similar to Eq. (26), holds between the different orders of $w$,

$$
w^{m+1}\left(\tau \tau^{\prime}\right)=-v_{c c k l} \int_{\tau^{\prime}}^{\tau} d \tau^{\prime \prime} \frac{\delta w^{m}\left(\tau \tau^{\prime \prime} ; u\right)}{\delta u_{k l}\left(\tau^{\prime+}\right)},
$$

where $\tau>\tau^{\prime}$, and $\tau$ is the time that refers to the variations of the density. The interaction $-v_{c c k l}$ couples the core level from one side to the variations of the external potential taken with valence levels on the other side. The negative sign can be understood in terms of the attractive core-hole charge, and $w^{m}$ can also be viewed in terms of the orders of an expansion to this core-hole potential. Therefore, the full matrix $w\left(\tau \tau^{\prime}\right)=\sum_{m}^{n} w^{m}\left(\tau \tau^{\prime}\right)$ plays the role of an effective interaction, accounting for all orders of the density variations due to the propagation of a core hole.

\section{B. Induced density variations}

In order to enable further interpretation and practical use, it is convenient to expand Eq. (28) for $m>0$ :

$$
\begin{aligned}
w^{m}\left(\tau \tau^{\prime}\right)= & (-1)^{m} v_{c c k_{1} l_{1}} \cdots v_{c c k_{m} l_{m}} \int_{\tau^{\prime}}^{\tau} \cdots \int_{\tau_{m-1}}^{\tau} d \tau_{1} \cdots \\
& \times d \tau_{m} \frac{\delta^{m} w^{0}\left(\tau \tau_{m} ; u\right)}{\delta u_{k_{1} l_{1}}\left(\tau^{\prime+}\right) \delta u_{k_{2} l_{2}}\left(\tau_{1}^{+}\right) \cdots \delta u_{k_{m} l_{m}}\left(\tau_{m-1}^{+}\right)} .
\end{aligned}
$$

With $w^{0}\left(\tau \tau^{\prime}\right)=W_{c}\left(\tau^{\prime} \tau\right)$, and keeping in mind that we are working with time-ordered response functions, we can write the cumulant as

$$
\begin{aligned}
C\left(t_{1} t_{2}\right)= & -i \int_{t_{1}}^{t_{2}} d \tau \int_{t_{1}}^{\tau} d \tau^{\prime} W_{c}\left(\tau, \tau^{\prime}\right) \\
& +i \int_{t_{1}}^{t_{2}} d \tau \sum_{m=1}^{\infty} v_{c c k_{1} l_{1}} \cdots v_{c c k_{m} l_{m}} \\
& \times \frac{(-1)^{(m+1)}}{(m+1) !} \int_{t_{1}}^{\tau} d \tau^{\prime} \cdots \int_{t_{1}}^{\tau} d \tau_{m} \\
& \times \frac{\delta^{m} W_{c}\left(\tau, \tau_{m}\right)}{\delta u_{k_{1} l_{1}}\left(\tau^{\prime}\right) \delta u_{k_{2} l_{2}}\left(\tau_{1}\right) \cdots \delta u_{k_{m} l_{m}}\left(\tau_{m-1}\right)},
\end{aligned}
$$

where the factor $1 /(m+1)$ ! is due to the extension of the integration domain, and repeated indices are summed over. Here, we have used the symmetry of the time-ordered screened interaction $W_{c}\left(\tau_{m}, \tau\right)=W_{c}\left(\tau, \tau_{m}\right)$. In order to obtain a practical formula, where higher orders are summed up, we have to make a connection with the density response. To this aim, we start by calculating a related cumulant function $C_{\mathrm{CDR}}\left(t_{1} t_{2}\right)$, which is obtained by replacing the time-ordered response functions in the above equation for $C$ with their retarded counterparts according to causal density response (CDR). The series can now be summed and produces two terms: The first yields the exchange correction to the energy of the core level while the rest of the sum is an expansion of the Coulomb potential acting on the core hole, created by the change in density $\Delta n$ at time $\tau$, which is in turn due to the potential $-v_{c c}(\mathbf{r}) \theta\left(t-t_{1}\right)$ created by the switching on of a core hole at time $t_{1}$. This potential is then integrated in $\tau$ over the time of propagation of the core hole. The compact result can be written as

$$
C_{\mathrm{CDR}}\left(t_{1} t_{2}\right)=-i v_{c c c c}\left(t_{2}-t_{1}\right)+\int_{t_{1}}^{t_{2}} d \tau v_{c c i j} \Delta n_{i j}\left(\tau-t_{1}\right) .
$$

However, $C_{\mathrm{CDR}} \neq C$, and simply replacing $C$ by $C_{\mathrm{CDR}}$, i.e., replacing time-ordered with causal response functions, leads to negative spectral weight on the high-energy side of the quasiparticle peak. This can be understood in the linearresponse limit, where Eq. (23) can be written as

$$
\begin{aligned}
C^{0}\left(t_{1} t_{2}\right)= & -i v_{c c c c}\left(t_{2}-t_{1}\right)+\int \frac{d \omega}{2 \pi} v_{c c i j} v_{c c k l} \frac{\chi_{i j k l}(\omega)}{\omega^{2}} f\left(\omega, t_{2}-t_{1}\right) \\
= & -i v_{c c c c}\left(t_{2}-t_{1}\right) \\
& -\int_{0}^{\infty} \frac{d \omega}{\pi} v_{c c i j} v_{c c k l} \frac{\operatorname{Im} \chi_{i j k l}(\omega)}{\omega^{2}} f\left(\omega, t_{2}-t_{1}\right),
\end{aligned}
$$


with $f(\omega, t) \equiv\left(e^{-i \omega t}+i \omega t-1\right)$. Note that here $\chi$ is the timeordered linear-response function. This leads to the inclusion of only positive frequencies in the last term in Eq. (32), while the use of a causal-response function has no restriction on the frequencies. Equation (32) can also be linked to the Fourier transform of the linear-response-induced density $\Delta n_{i j}^{0}$,

$$
\operatorname{Re} \Delta n_{i j}^{0}(\omega)=-\frac{\operatorname{Im} \chi_{i j k l}(\omega)}{\omega} v_{c c k l}
$$

so that

$$
\begin{aligned}
C^{0}\left(t_{1} t_{2}\right)= & -i v_{c c c c}\left(t_{2}-t_{1}\right) \\
& +\int_{0}^{\infty} \frac{d \omega}{\pi} \frac{v_{c c i j} \operatorname{Re} \Delta n_{i j}^{0}(\omega)}{\omega} f\left(\omega, t_{2}-t_{1}\right) .
\end{aligned}
$$

Together with Eq. (31), this suggests an approximation for the full cumulant to all orders; namely, we substitute the full induced density $\Delta n$ in place of the linear response $\Delta n^{0}$. This finally yields the approximate cumulant

$$
\begin{aligned}
C_{\mathrm{TDR}}\left(t_{1} t_{2}\right)= & -i v_{c c c c}\left(t_{2}-t_{1}\right)+\int_{0}^{\infty} \frac{d \omega}{\pi} \frac{v_{c c i j} \operatorname{Re}\left[\Delta n_{i j}(\omega)\right]}{\omega} \\
& \times\left[e^{-i \omega\left(t_{2}-t_{1}\right)}+i \omega\left(t_{2}-t_{1}\right)-1\right]
\end{aligned}
$$

where TDR stands for time-ordered density response [58], which only includes positive frequency components. This result has a physical interpretation which moreover has a significant practical advantage: since the core orbital is kept fixed the perturbation is known and its effect can be calculated directly. For example, the TDDFT in real space and time can be used, as proposed in Ref. [59] for the case of linear response. It is then not necessary to calculate the rather complicated higher-order response functions, which thereby overcomes the issues discussed in Ref. [5].

When the core hole is suddenly switched on, the system may react violently. However, an interacting system will eventually reach a new equilibrium, given by the final state of the system with a static core hole. In this limit $\Delta n_{i j}(\tau)$ becomes independent of $\tau$. The simplest approximation is therefore a shift of the core level due to the fact that the valence density should be calculated in the presence of the core hole. Since this approximation completely neglects dynamical effects, it cannot lead to satellites in the spectral function. In order to do better, one could apply the same reasoning starting from the next order, which means, in practice, to evaluate explicitly the lowest-order cumulant with the valence density calculated in the presence of the core hole. Such an intuitive approach (which has been used successfully, e.g., in Refs. [60-62]) may be justified by our derivation.

\section{Self-energy}

Let us now compare the above result with what one would obtain in the context of a Dyson equation. For this case, we start again from the exact expression in Eq. (5) and then, using $\delta G / \delta u^{H}=-G\left(\delta G^{-1} / \delta u^{H}\right) G$, the exact Dyson equation becomes

$$
\begin{aligned}
G(12)= & G^{H}(12)-i G^{H}(1 \overline{1}) W(\overline{1} \overline{4} ; u) \\
& \times G(\overline{1} \overline{5}) \frac{\delta G^{-1}(\overline{5} \overline{6})}{\delta u^{H}\left(\overline{4}^{+}\right)} G(\overline{6} 2),
\end{aligned}
$$

which has a self-energy given by

$$
\begin{aligned}
\Sigma_{\mathrm{xc}}(16)= & -i W(1 \overline{4} ; u) G(1 \overline{5}) \frac{\delta G^{-1}(\overline{5} 6)}{\delta u^{H}\left(\overline{4}^{+}\right)} \\
= & i W(1 \overline{4} ; u) G(1 \overline{5}) \\
& \times\left[\delta(\overline{5} 6) \delta(\overline{5} \overline{4})+\frac{\delta \Sigma_{\mathrm{xc}}(\overline{5} 6)}{\delta u^{H}\left(\overline{4}^{+}\right)}\right] .
\end{aligned}
$$

Again we take the matrix element in the core orbital and make the same approximations as throughout the above derivations. For $t_{6}>t_{1}$ this allows us to make the ansatz $\Sigma_{\mathrm{xc}}\left(t_{1} t_{6}\right) \equiv$ $i G_{c c}\left(t_{1} t_{6}\right) w^{\text {eff }}\left(t_{1} t_{6}\right)$. If one neglects the variations of $G_{c c}$ with respect to $u$, we find

$$
\begin{aligned}
& G_{c c}\left(t_{1} t_{6}\right) w^{\mathrm{eff}}\left(t_{1} t_{6}\right) \\
&= i W_{c}\left(t_{1} \bar{t}_{6} ; u\right) G_{c c}\left(t_{1} \bar{t}_{6}\right) \\
& \quad+i W_{c}\left(t_{1} \bar{t}_{4} ; u\right) G_{c c}\left(t_{1} \bar{t}_{5}\right) G_{c c}\left(\bar{t}_{5} t_{6}\right) \frac{\delta w^{\mathrm{eff}}\left(\bar{t}_{5} t_{6}\right)}{\delta u^{H}\left(\bar{t}_{4}^{+}\right)} .
\end{aligned}
$$

Then using the relation

$$
\int_{-\infty}^{+\infty} d \bar{t}_{5} G_{c c}\left(t_{1} \bar{t}_{5}\right) G_{c c}\left(\bar{t}_{5} t_{6}\right)=i G_{c c}\left(t_{1} t_{6}\right) \int_{t_{1}}^{t_{6}} d \bar{t}_{5}
$$

the core-level self-energy becomes

$$
\begin{aligned}
\Sigma_{\mathrm{xc}}\left(t_{1} t_{6}\right)= & i G_{c c}\left(t_{1} t_{6}\right)\left[W_{c}\left(t_{1} t_{6} ; u\right)\right. \\
& \left.+i W_{c}\left(t_{1} \bar{t}_{4} ; u\right) \int_{t_{1}}^{t_{6}} d \bar{t}_{5} \frac{\delta w^{\mathrm{eff}}\left(\bar{t}_{5} t_{6}\right)}{\delta u^{H}\left(\bar{t}_{4}^{+}\right)}\right] .
\end{aligned}
$$

This self-energy has the same structure as the GWA, but instead of a linear response $W$, one has an effective interaction

$$
\begin{aligned}
w^{\mathrm{eff}}\left(t_{1} t_{6} ; u\right) \equiv & W_{c}\left(t_{1} t_{6} ; u\right)+i W_{c}\left(t_{1} \bar{t}_{4} ; u\right) \\
& \times \int_{t_{1}}^{t_{6}} d \bar{t}_{5} \frac{\delta w^{\mathrm{eff}}\left(\bar{t}_{5} t_{6}\right)}{\delta u^{H}\left(\bar{t}_{4}^{+}\right)} .
\end{aligned}
$$

This result is identical to the effective interaction that appears in the cumulant, $w^{\text {eff }}=w$, with $w$ defined in Eq. (28). The variation of $G_{c c}$ with respect to $u$, which is neglected in this result, does not contribute to first order in $W_{c}$, but modifies $w^{\text {eff }}$ starting from second order, where it adds a contribution $G_{c c}\left(t_{1} \bar{t}_{5}\right) W_{c}\left(t_{1}, \bar{t}_{4}^{+}\right) G_{c c}\left(\bar{t}_{5} \bar{t}_{4}^{+}\right) G_{c c}\left(\bar{t}_{4}^{+} t_{6}\right) W_{c}\left(\bar{t}_{5} t_{6}\right)$ to the self-energy. This is the reason why the GW self-energy and the lowest-order cumulant have the same effective interaction, namely, the linear-response screened Coulomb interaction $W$. For the higher orders, it is instead important that the effective interactions are different, since $w$ has to be used in the cumulant and $w^{\text {eff }}$ in the Dyson equation, while both are in principle exact within the approximations made here. For example, the extra second-order term that appears in the self-energy for the Dyson equation is not needed in $w^{\text {eff }}$ since it is automatically included in the cumulant Green's function. 
Finally, the form $\Sigma_{\mathrm{xc}}=i G w^{\text {eff }}$ is reminiscent of the $T$ matrix formalism [43,63]. Such $T$-matrix approximations can be derived by supposing that the self-energy consists of a Green's function and an effective interaction which, inserted into Eq. (37), allow one to derive a Dyson equation for the effective interaction. However, for our present core-hole problem we have assumed that the core Green's function does not vary though derivatives of the interaction are taken into account. Instead, to obtain the scattering diagrams in the $T$-matrix approximations the Green's function is varied and the effective interaction is kept fixed [43,63]. This is more important in a situation of low density (partial filling), which is quite different from the core-hole problem.

\section{MODEL CALCULATIONS}

As a concrete illustration of the approach presented here, we apply it to a simple three-state model, similar to that used by Lee, Gunnarsson, and Hedin in Ref. [64]. This model has also been used to treat charge-transfer satellites in x-ray spectra [65], and similar models have been used in other contexts [66-68]. This model system with two electrons, a core electron and a valence electron propagating in two atomic levels $a, b$, is described by the Hamiltonian

$$
\hat{H}=\epsilon_{0} \hat{c}^{\dagger} \hat{c}+\epsilon_{a}^{0} \hat{n}_{a}+\epsilon_{b}^{0} \hat{n}_{b}-U \hat{n}_{h} \hat{n}_{a}-t\left(\hat{c}_{a}^{\dagger} \hat{c}_{b}+\hat{c}_{b}^{\dagger} \hat{c}_{a}\right),
$$

where $\epsilon^{0}$ are atomic energies evaluated in the presence of the core electron, and $U$ is the potential from the core hole $\hat{n}_{h}=1-\hat{n}_{c}$ coupling only to one of the two levels, $a$. The valence levels belong to different atoms and this justifies the approximation of weak coupling to the level $b$. The hybridization between levels $a$ and $b$ is represented by the interaction parameter $t$. This model aids a physical understanding of core photoemission in molecules with flat valence bands, where charge transfer excitations between different atoms modify the spectrum.

The initial state, where $n_{h}=0$, is described by the twoparticle state $\left|\psi_{0}^{i}\right\rangle=\sin \phi|a\rangle|c\rangle+\cos \phi|b\rangle|c\rangle$ that mixes core and valence levels, with $\tan 2 \phi=2 t / \epsilon$ and $\epsilon=\epsilon_{a}^{0}-\epsilon_{b}^{0}$. The energy of the initial state is given by $\epsilon_{1,0}^{i}=\frac{1}{2}\left(\epsilon_{a}^{0}+\right.$ $\left.\epsilon_{b}^{0}\right) \pm \frac{1}{2} \sqrt{\epsilon^{2}+4 t^{2}}$. The final states where $n_{h}=1$ have only one particle and are given by the single-particle wavefunctions $\left|\Psi_{1}^{f}\right\rangle=\cos \theta|a\rangle-\sin \theta|b\rangle$ and $\left|\Psi_{2}^{f}\right\rangle=\sin \theta|a\rangle+$ $\cos \theta|b\rangle$, with $\tan 2 \theta=2 t /(\epsilon-U)$ and $\epsilon_{1,2}^{f}=\frac{1}{2}\left(\epsilon_{a}^{0}+\epsilon_{b}^{0}-\right.$ $U) \pm \frac{1}{2} \sqrt{(\epsilon-U)^{2}+4 t^{2}}$. In the model the core-hole potential couples only to level $a$ and therefore the interaction with the time-dependent occupation of level $a$ will appear.

In order to obtain the cumulant solution for this model, we apply Eq. (30):

$$
\begin{aligned}
C\left(t_{1} t_{2}\right)= & -i U n_{a}^{0}\left(t_{2}-t_{1}\right)+\left.i U^{2} \int_{t_{1}}^{t_{2}} d \tau \int_{t_{1}}^{\tau} d \tau^{\prime} \chi\left(\tau, \tau^{\prime} ; u_{a}\right)\right|_{u_{a}=0} \\
& -\left.i \frac{U^{3}}{2} \int_{t_{1}}^{t_{2}} d \tau \int_{t_{1}}^{\tau} d \tau^{\prime} \int_{t_{1}}^{\tau} d \tau_{1} \frac{\delta \chi\left(\tau, \tau_{1}\right)}{\delta u_{a}\left(\tau^{\prime}\right)}\right|_{u_{a}=0} \cdots,
\end{aligned}
$$

where $n_{a}^{0}$ is the occupation of state $a$ in the ground state, and the first term, linear in $U$, replaces the bare Coulomb interaction seen in Eq. (35) for the model Hamiltonian, and causes an overall quasiparticle shift.

Finally we apply Eq. (35) for the cumulant, which yields the TDR approximation

$$
\begin{aligned}
C_{\mathrm{TDR}}\left(t_{1} t_{2}\right)= & i U n_{a}^{0}\left(t_{2}-t_{1}\right)+U \int_{0}^{\infty} \frac{d \omega}{\pi} \frac{\operatorname{Re}\left[\Delta n_{a}(\omega)\right]}{\omega} \\
& \times\left[e^{-i \omega\left(t_{2}-t_{1}\right)}+i \omega\left(t_{2}-t_{1}\right)-1\right]
\end{aligned}
$$

where the density is given in terms of the time-dependent wavefunction, $n_{a}(t)=|\langle a \mid \psi(t)\rangle|^{2}$, and $|\psi(t)\rangle$ is the state of the system at time $t$ after the appearance of the core hole, and is initially equal to the ground-state valence wavefunction, $|\psi(0)\rangle=\left|\psi_{0}^{i}\right\rangle$. We can now compare the results obtained from the two lowest orders of Eq. (43), the CDR approximation Eq. (31), and the TDR approximation Eq. (44).

Results for the core-level spectral function $A_{c}(\omega)=$ $-(1 / \pi) \operatorname{Im} G(\omega)$ vs energy $\omega-\epsilon_{c}$ are shown in Fig. 1. The position of the peaks reflects the energy of the transition of the valence electron from the initial (with the core electron) to the final (with the core hole) state, while the height of the peaks corresponds to the probability amplitude of the transition. Only a non-negligible value of the parameter $t$ allows for the core potential to affect the transition energies between the initial and the final states, since otherwise no screening can happen. From top to bottom, the curves show results for core-hole strength $U=1$ (top), $U=7$ (middle), and $U=20$ (bottom), corresponding to weak, intermediate, and strong coupling, all with the parameters $t=3$ and $\epsilon=1$. The exact results, as well as those from the TDR approximation in Eq. (44), show the same general trends as previous solutions of a similar model Hamiltonian which coupled an adsorbate state to a partially occupied band [68], with growth of the satellite splitting and amplitude as a function of the core-hole strength $U$. The top set of curves represents the weak-coupling limit, which can be seen by the lack of any visible satellite, and by the agreement of all curves, which should be nearly identical in the linear-response regime. At intermediate coupling ( $U=7$, middle), the various approximations now give appreciably different results. The linear response approximation (green) underestimates the splitting between the satellite and quasiparticle positions by nearly a factor of 2, underestimates the size of the quasiparticle peak, and produces a second satellite, indicating a bosonlike progression, as expected. The lowest-order $\left(U^{3}\right)$ corrected result (purple) gives a small correction to the quasiparticle weight and position, but does little to correct the splitting between the quasiparticle and satellite. The issues with the linear response and $U^{3}$ corrected approximations become even more apparent in the strong-coupling regime $(U=20)$, where the linear response greatly underestimates quasiparticle weight and quasiparticle-satellite splitting, and produces a long progression of satellites, initially with increasing weight, and overestimates the quasiparticle energy by a large amount. This is unphysical, since a single valence electron cannot produce multiple excitations. Also the inclusion of the $U^{3}$ 


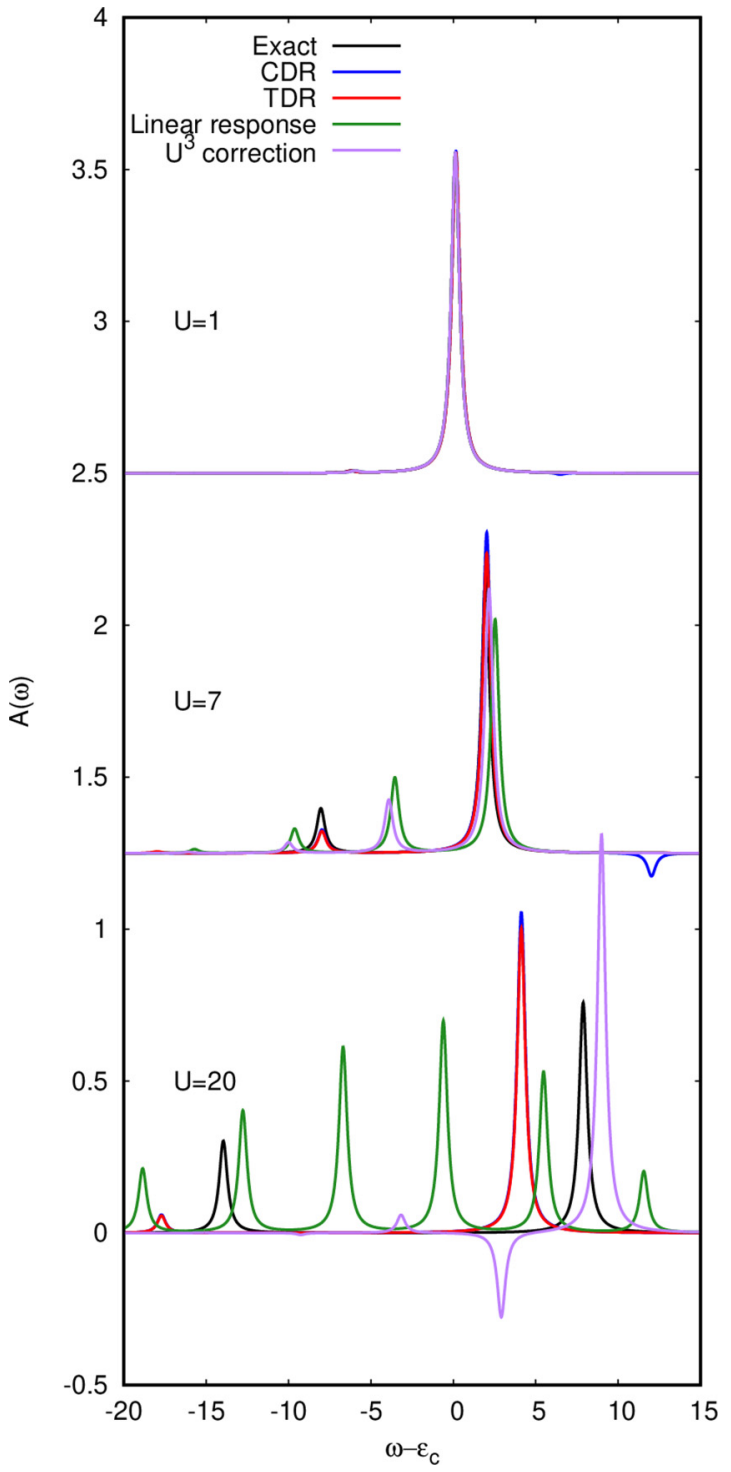

FIG. 1. Spectral function $A(\omega)=(-1 / \pi) \operatorname{Im} G_{c}(\omega)$ vs energy $\omega-\epsilon_{c}$ for model parameters $\epsilon=1 \mathrm{eV}$ and $t=3 \mathrm{eV}$. The couplings $U=1,3$, and 7 simulate weak, intermediate, and strong coupling to the core hole, respectively. Results are shown for the exact spectral function (black), the CDR approximation (blue), the TDR approximation (red), the linear-response approximation (green), and from including the first nonlinear $\left(U^{3}\right)$ correction term (purple). In the exact solution, the main peak and satellite positions are given by the eigenvalues of the single-particle Hamiltonian in the presence of the core hole, while in the linear cumulant, they are given by an expansion of the same energies to linear order in $U$. Note that the results from the CDR and TDR approximations are in good agreement for the positions of the quasiparticle and satellite peaks for $U=1$ and $U=7$, and even at $U=20$ the excitation energy (difference between quasiparticle and satellite) matches the exact result. However, the CDR approximation produces small negative satellites on the high-energy side of the quasiparticle peak. In contrast, the linear response and $U^{3}$ corrected approximations greatly underestimate the satellite energy and give unphysical results such as a large bosonlike satellite progression and negative spectral weight. correction produces spurious negative spectral weight. In contrast, the TDR approximation [Eq. (35)] produces results in good agreement for the quasiparticle and satellite peak positions for $U=7$, and even reproduces the satellite splitting at $U=20$, although the satellite weight is underestimated in both cases, while the CDR approximation produces negative spectral weight above the quasiparticle peak. The intuitive TDR approximation given by Eq. (35) corrects this unphysical behavior by filtering out the negative frequency components of the induced density. Results for the TDR approximation (red) show little difference in comparison to those obtained with the CDR, apart from the lack of any negative spectral weight, demonstrating that this approximation should be a reasonable method for obtaining physical results that include nonlinear corrections.

\section{CONCLUSIONS}

We have demonstrated that the Kadanoff-Baym functional differential equation is a convenient starting point to derive the form of the cumulant Green's function beyond the linearresponse approximation. For a single level, such as a localized deep core level that can be considered decoupled from the rest of the system, the result can be approximated in a compact way, which highlights the essential physics: the sudden switching on of a core hole perturbs the valence density, and the subsequent time integral of the change in density leads to a quasiparticle correction and to satellites in the spectral function. The approach, which we term the time-ordered density response or the TDR approximation, is tested on a simple three-level model system similar to that of Lee, Gunnarsson, and Hedin. The numerical results suggest that molecular systems with strong nonlinear core-hole effects can significantly improve the calculated photoemission spectra and generally need to be taken into account. We suggest that coupling this nonlinear cumulant approach with real-time TDDFT can be a promising way to include the nonlinear effects in $a b$ initio calculations of the core-level photoemission spectra for such systems.

\section{ACKNOWLEDGMENTS}

This work was carried out with support from the U.S. Department of Energy, Office of Science, BES, Chemical Sciences, Geosciences and Biosciences Division in the Center for Scalable and Predictive methods for Excitations and Correlated phenomena (SPEC) at PNNL, and with computer support from the U.S. DOE NERSC and from GENCI (544). L.R., M.T., and J.J.R. also acknowledge support at the beginning of this project from the European Research Council (ERC Grant Agreement No. 320971). 
[1] L. Hedin, J. Phys.: Condens. Matter 11, R489 (1999).

[2] G. Wentzel, Z. Phys. 31, 445 (1925).

[3] G. Wentzel, Z. Phys. 34, 730 (1925).

[4] R. D. Richtmyer, Phys. Rev. 49, 1 (1936).

[5] G. D. Mahan, Phys. Rev. B 25, 5021 (1982).

[6] B. Roulet, J. Gavouret, and P. Nozières, Phys. Rev. 178, 1072 (1969).

[7] P. Nozières, J. Gavouret, and B. Roulet, Phys. Rev. 178, 1084 (1969).

[8] P. Nozières and C. T. De Dominicis, Phys. Rev. 178, 1097 (1969).

[9] J. N. Andersen and C.-O. Almbladh, J. Phys.: Condens. Matter 13, 11267 (2001).

[10] D. C. Langreth, Phys. Rev. 182, 973 (1969).

[11] U. von Barth and G. Grossmann, Phys. Rev. B 25, 5150 (1982).

[12] B. Lundqvist, Phys. Kondens. Materie 9, 236 (1969).

[13] L. Hedin, J. Michiels, and J. Inglesfield, Phys. Rev. B 58, 15565 (1998).

[14] D. Dunn, Can. J. Phys. 53, 321 (1975).

[15] O. Gunnarsson, V. Meden, and K. Schönhammer, Phys. Rev. B 50, 10462 (1994).

[16] C. Verdi, F. Caruso, and F. Giustino, Nat. Commun. 8, 15769 (2017).

[17] G. D. Mahan, Phys. Rev. 145, 602 (1966).

[18] D. C. Langreth, Phys. Rev. B 1, 471 (1970).

[19] C. O. Almbladh and P. Minnhagen, Phys. Rev. B 17, 929 (1978).

[20] M. Cini and A. D’Andrea, J. Phys. C: Solid State Physics 21, 193 (1988).

[21] J.-J. Chang and D. C. Langreth, Phys. Rev. B 5, 3512 (1972).

[22] J.-J. Chang and D. C. Langreth, Phys. Rev. B 8, 4638 (1973).

[23] F. Bechstedt, Phys. Status Solidi B 101, 275 (1980).

[24] F. Bechstedt, R. Enderlein, and M. Koch, Phys. Status Solidi B 99, 61 (1980).

[25] S. Huefner, Photoemission Spectroscopy: Principles and Applications (Springer-Verlag, Berlin, 2003).

[26] F. De Groot and A. Kotani, Core Level Spectroscopy of Solids, Advances in Condensed Matter Science (Taylor \& Francis, Philadelphia, 2008).

[27] G. Mahan, Many-Particle Physics (Plenum Press, New York, 1990).

[28] J. P. Nery, P. B. Allen, G. Antonius, L. Reining, A. Miglio, and X. Gonze, Phys. Rev. B 97, 115145 (2018).

[29] F. Aryasetiawan, L. Hedin, and K. Karlsson, Phys. Rev. Lett. 77, 2268 (1996).

[30] M. Vos, A. S. Kheifets, E. Weigold, S. A. Canney, B. Holm, F. Aryasetiawan, and K. Karlsson, J. Phys.: Condens. Matter 11, 3645 (1999).

[31] A. S. Kheifets, V. A. Sashin, M. Vos, E. Weigold, and F. Aryasetiawan, Phys. Rev. B 68, 233205 (2003).

[32] M. Guzzo, G. Lani, F. Sottile, P. Romaniello, M. Gatti, J. J. Kas, J. J. Rehr, M. G. Silly, F. Sirotti, and L. Reining, Phys. Rev. Lett. 107, 166401 (2011).

[33] M. Gatti and M. Guzzo, Phys. Rev. B 87, 155147 (2013).

[34] J. Lischner, D. Vigil-Fowler, and S. G. Louie, Phys. Rev. Lett. 110, 146801 (2013).

[35] M. Guzzo, J. J. Kas, L. Sponza, C. Giorgetti, F. Sottile, D. Pierucci, M. G. Silly, F. Sirotti, J. J. Rehr, and L. Reining, Phys. Rev. B 89, 085425 (2014).
[36] J. S. Zhou, J. J. Kas, L. Sponza, I. Reshetnyak, M. Guzzo, C. Giorgetti, M. Gatti, F. Sottile, J. J. Rehr, and L. Reining, J. Chem. Phys. 143, 184109 (2015).

[37] J. Lischner, G. K. Pálsson, D. Vigil-Fowler, S. Nemsak, J. Avila, M. C. Asensio, C. S. Fadley, and S. G. Louie, Phys. Rev. B 91, 205113 (2015).

[38] K. Nakamura, Y. Nohara, Y. Yosimoto, and Y. Nomura, Phys. Rev. B 93, 085124 (2016).

[39] B. Gumhalter, V. Kovač, F. Caruso, H. Lambert, and F. Giustino, Phys. Rev. B 94, 035103 (2016).

[40] F. Caruso and F. Giustino, Phys. Rev. B 94, 115208 (2016).

[41] A. L. Fetter and J. D. Walecka, Quantum Theory of ManyParticle Systems (McGraw-Hill, New York, 1971).

[42] L. Hedin, Phys. Rev. 139, A796 (1965).

[43] R. Martin, L. Reining, and D. Ceperley, Interacting Electrons: Theory and Computational Approaches (Cambridge University Press, Cambridge, UK, 2016).

[44] D. C. Langreth, Collective Properties of Physical Systems (Elsevier, Amsterdam, 1973), pp. 210-222.

[45] P. Minhagen, Phys. Lett. A 56, 327 (1976).

[46] W. Hänsch and P. Minnhagen, Phys. Rev. B 26, 2772 (1982).

[47] B. Gumhalter, Phys. Rev. B 72, 165406 (2005).

[48] Y. Pavlyukh, Sci. Rep. 7, 504 (2017).

[49] S. Mukamel, Phys. Rev. B 72, 235110 (2005).

[50] A. Marini and Y. Pavlyukh, Phys. Rev. B 98, 075105 (2018).

[51] J. J. Rehr, F. D. Vila, J. J. Kas, N. Y. Hirshberg, K. Kowalski, and B. Peng, J. Chem. Phys. 152, 174113 (2020).

[52] F. Vila, J. Rehr, J. Kas, B. Peng, and K. Kowalski, arXiv:2006.07477.

[53] E. Runge and E. K. U. Gross, Phys. Rev. Lett. 52, 997 (1984).

[54] P. C. Martin and J. Schwinger, Phys. Rev. 115, 1342 (1959).

[55] G. Baym and L. P. Kadanoff, Phys. Rev. 124, 287 (1961).

[56] G. Lani, P. Romaniello, and L. Reining, New J. Phys. 14, 013056 (2012).

[57] G. Stefanucci and R. van Leeuwen, Nonequilibrium Many-Body Theory of Quantum Systems: A Modern Introduction, 1st ed. (Cambridge University Press, Cambridge, UK, 2013).

[58] J. J. Kas, F. D. Vila, J. J. Rehr, and S. A. Chambers, Phys. Rev. B 91, 121112(R) (2015).

[59] J. J. Kas, J. J. Rehr, and J. B. Curtis, Phys. Rev. B 94, 035156 (2016).

[60] R. Pandey and S. Mukamel, J. Chem. Phys. 124, 094106 (2006).

[61] B. Brena, S. Carniato, and Y. Luo, J. Chem. Phys. 122, 184316 (2005).

[62] B. Brena, Y. Luo, M. Nyberg, S. Carniato, K. Nilson, Y. Alfredsson, J. Åhlund, N. Mårtensson, H. Siegbahn, and C. Puglia, Phys. Rev. B 70, 195214 (2004).

[63] P. Romaniello, F. Bechstedt, and L. Reining, Phys. Rev. B 85, 155131 (2012).

[64] J. D. Lee, O. Gunnarsson, and L. Hedin, Phys. Rev. B 60, 8034 (1999).

[65] E. Klevak, J. J. Kas, and J. J. Rehr, Phys. Rev. B 89, 085123 (2014).

[66] B. Gumhalter, J. Phys. C: Solid State Phys. 10, L219 (1977).

[67] K. Schonhammer and O. Gunnarsson, Solid State Commun. 23, 691 (1977).

[68] O. Gunnarsson and K. Schönhammer, Phys. Rev. Lett. 41, 1608 (1978). 